\title{
Evaluación del Software Educativo Mundo Agroforestal con Jóvenes Rurales de Nariño, Colombia
}

\author{
Yesenia A. Narvaez-Romo, Gloria C. Luna, Hugo F. Leonel y Jaime O. Ruiz \\ Universidad de Nariño, Facultad de Ciencias Agrícolas, Programa de Ingeniería Agroforestal e Ingeniería \\ Electrónica. Calle 18 Carrera 50 Ciudadela Universitaria Torobajo, Pasto, Nariño, Colombia. \\ (e-mail: nrale.14@gmail.com; grupopifil@gmail.com; hleonel2001@gmail.com; jaimeruiz.pasto@hotmail.com)
}

Recibido Jun. 30, 2016; Aceptado Sep. 5, 2016; Versión final Nov. 1, 2016, Publicado Abr. 2017

\begin{abstract}
Resumen
El objetivo de esta investigación fue evaluar el software educativo Mundo Agroforestal con jóvenes rurales de la Institución Educativa Francisco de la Villota en Nariño, Colombia. Las Tecnologías de la Información y Comunicación son un recurso usado para facilitar los procesos de formación. El software educativo Mundo Agroforestal dada su reciente etapa de desarrollo no ha sido evaluado en cuanto a la relación de los aprendizajes que se adquieren por medio de éste. Se realizó el análisis con T de Student y modelo lineal general. Se obtuvieron en el tiempo aprendizajes significativos en agroforestería. Se observó que el uso del software facilitó la comprensión de las diferentes temáticas, generando motivación e interés en la exploración de las interfaces para aprovechar esta herramienta en un espacio de interacción dinámico mejorando el autoaprendizaje.
\end{abstract}

Palabras clave: agroforestal; enseñanza; aprendizaje; jóvenes rurales; software educativo

\section{Evaluation of the Educational Software Agroforestry World (Mundo Agroforestal) with Rural Youth of Nariño, Colombia}

\begin{abstract}
The objective of the study presented in this paper was evaluating the educational software Agroforestry World (Mundo Agroforestal) by rural young men and women from the educational institution Francisco de la Villota in Nariño, Colombia. Information and Communication Technologies are widely used for facilitating educational processes. The relatively new educational software Agroforestry World has not been assessed regarding the learning outcomes that might result from its application. Analyses were done through a TStudent test and a general linear model. After some time, significant learning processes in agroforestry were obtained. It was observed that the use of the software facilitated students' comprehension of the different topics, improving self-learning.
\end{abstract}

Keywords: agroforestry; teaching; learning; rural youth; educational software 


\section{INTRODUCCIÓN}

Los procesos de enseñanza y aprendizaje, tienen como propósito contribuir a la formación del estudiante; para el desarrollo de este proceso, se hace necesario utilizar diferentes procedimientos o instrumentos de apoyo comúnmente llamados medios de enseñanza (De la Torre y Domínguez, 2012). Los medios de enseñanza se agrupan de manera general en medios de percepción directa, imágenes fijas y en movimiento, sonido, situación real y simulación, así como los apoyados en el uso de las tecnologías de la información y la comunicación -TIC (Cabero, 2015).

Las tecnologías de la información y la comunicación-TIC, se han promocionado y fortalecido potencialmente para lograr cambios educativos, es así como se vienen formulando nuevas alternativas de enseñanza y aprendizaje, orientadas a transmitir el conocimiento de manera dinámica (Saidu et al., 2014); caracterizándose como una herramienta didáctica que favorece la enseñanza, comprensión y comunicación, proporcionando soluciones con impacto directo en el tejido social; debido a que los entornos multimedia se ajustan de manera versátil, dinámica e interactiva a las necesidades de la comunidad en pro de mediatizar el conocimiento en un escenario de globalización de la información (Builes, 2016).

También las TIC han cambiado la forma de vida de los seres humanos, en el sector agrícola la transformación de la producción y comercialización, se debe en parte a la incorporación de las TIC en los procesos, para mejorar la competitividad y optimizar la producción, por medio del acceso oportuno a la información (Gómez et al., 2016); logrando importantes resultados en el acercamiento del conocimiento a comunidades rurales, y convirtiéndose en canales de comunicación que apoyan el intercambio de información veraz, útil, oportuna y significativa en los procesos de enseñanza - aprendizaje (Cox et al., 2013; Bossio et al., 2005; Leonel y Luna, 2016).

Los software educativos, son aplicaciones o programas de computación que se utilizan como herramienta de apoyo, valiosa e imprescindible en el proceso de enseñanza aprendizaje (Clavera et al., 2015); por lo tanto, se han adelantado procesos investigativos en la evaluación de software educativos en temáticas, como: ciencias ambientales, lenguaje, matemáticas, entre otras (Ledo et al., 2010); sin embargo, su aplicación en agroforestería, aún es mínimo, debido al difícil acceso de tecnologías comunicacionales por parte de las comunidades rurales (León et al., 2014).

En Colombia mediante la Ley 1341 de 2009, se crea la Agencia Nacional Espectro que define los principios y conceptos sobre la sociedad de la información y la organización de las tecnologías de la información y las comunicaciones, haciendo referencia al conjunto de recursos, herramientas, equipos, programas informáticos, aplicaciones, redes y medios, para la compilación, procesamiento, almacenamiento y transmisión de información. De esta manera se promueve a lo largo del territorio nacional el acceso a los llamados "Puntos digitales" a fin de promover el conocimiento, obtener habilidades y destrezas en estos aspectos tecnológicos.

Ante esta situación, contar con un software educativo agroforestal para el sector rural, permite ampliar cobertura en los procesos de educación pertinente, dado que existe necesidad de materiales que promuevan la formación integral, enfocados a sistemas productivos sustentables; integrando apropiación e innovación en un mismo plano, que se dan a partir de las sinergias entre sectores académicos, productivos y estatales como propuesta para alcanzar la apropiación social del conocimiento (Leonel y Luna, 2016).

Por lo anterior, el programa de Ingeniería Agroforestal de la Universidad de Nariño, mediante el grupo Plan de Investigación para el Fortalecimiento Integral de las Comunidades- PIFIL, diseñó, elaboró y validó interdisciplinariamente el software educativo "Mundo Agroforestal" dirigido a jóvenes rurales (León et al., 2014); el cual, dada su reciente etapa de desarrollo no ha sido evaluado en cuanto a la relación de los aprendizajes que se adquieren por medio de éste. El presente trabajo de investigación tuvo por objetivo evaluar el software educativo "Mundo Agroforestal", el cual se abordó al analizar la relación del aprendizaje adquirido entre grupos control y experimental, en el contexto de los jóvenes rurales de grados décimos y once de la Institución Educativa Francisco de la Villota.

\section{METODOLOGÍA}

El presente estudio se realizó en la Institución Educativa Francisco de la Villota, corregimiento de Genoy, vereda Nueva Campiña, ubicado a 2400 m.s.n.m, a $13 \mathrm{Km}$ de la ciudad de Pasto; con coordenadas $1^{\circ} 27^{\circ} 18^{\prime \prime}$ $\mathrm{N}$ y $77^{\circ} 33^{\prime} 8^{\prime \prime}$ 'W. Limita al norte con el municipio de Nariño, y el corregimiento de la Caldera, al sur con el corregimiento de Mapachico, al oriente con el corregimiento de Morasurco y al occidente con el municipio de Nariño. Se trabajó con el total de 64 jóvenes rurales de grados superiores $\left(10^{\circ}\right.$ y $\left.11^{\circ}\right)$ seleccionados por criterios como: disponibilidad de tiempo en las diferentes sesiones de trabajo, voluntad de participación y facilidad en el uso de tecnologías. La respectiva asignación de los estudiantes a los grupos experimental y control fue realizada por medio del muestreo aleatorio estratificado. 
La investigación tiene un enfoque cuantitativo correlacional, con diseño experimental tipo pre experimento pre post y grupo control (Hernández et al., 2007). Con el siguiente proceso metodológico: a) Revisión de información secundaria (León et al., 2014; Cova et al., 2008; Fossatti, 2009), y socialización del proyecto a la comunidad educativa, b) Levantamiento de información primaria: se diseñó y validó la evaluación de aprendizajes, con un grupo interdisciplinario de profesionales en agroforestería e informática, teniendo en cuenta los estándares básicos de competencias para los grados décimo y once, en relación con las temáticas desarrolladas en el software "Mundo Agroforestal, que permitió capacitar al grupo experimental y al grupo control por medio de charlas educativas con materiales didácticos manuales, c) Evaluaciones de aprendizajes: Éstas fueron realizadas antes y después de las capacitaciones en agroforestería, donde la naturaleza de los contenidos a evaluar es con preguntas de recuerdo. El primer momento se denominó evaluación diagnóstica (Ed) y el segundo evaluación posterior (Ep), se hizo la comparación (Ed y Ep) de las variables generales, que hacen referencia al conocimiento de agroforestería y se complementó la información con variables específicas, que solo se emplean en la evaluación posterior para aclarar la temática de arreglos agroforestales (Tabla 1), con tres repeticiones, que fueron: R1: Al terminar las capacitaciones; R2: Al segundo mes de terminadas las capacitaciones; y R3: Al cuarto mes de terminadas las capacitaciones.

Los resultados de cada evaluación se obtuvieron con el promedio de la suma de puntajes obtenidos en cada pregunta, para lo cual: en la evaluación diagnóstica se manejó la escala de 0 no tiene conocimiento, 3 tiene algún conocimiento y 5 tiene conocimiento. Para las evaluaciones posteriores se manejó la escala de 0 no tiene conocimiento y 1 tiene conocimiento. Para evaluar los resultados de cada estudiante, se empleó la escala de valoración desempeño bajo $(0,0-3,0)$ indicando la no aprobación, desempeño básico $(3,1-4,0)$, desempeño alto $(4,1-4,5)$ y desempeño superior $(4,6-5,0)$ indicando la aprobación de los contenidos en diferentes niveles de conocimiento. Lo anterior, establecido de acuerdo al Decreto 1290 de 2009 de Colombia y en la Institución Educativa Francisco de la Villota.

Para el análisis de los resultados estadísticos, se validaron los datos para pruebas paramétricas por medio de la normalidad (Shapiro-Wilks modificada), siendo asumida por la cantidad superior a 30 estudiantes y la homocedasticidad (Levene modificada). Se realizaron prueba $T$ Student con muestras independientes para observar diferencias estadísticas entre los grupos y evaluaciones generales y específicas. Se llevó a cabo un modelo lineal general con medidas repetidas de forma intrasujetos (evaluaciones generales y específicas) e intersujetos (grupos control y experimental) para analizar las evaluaciones en el tiempo con relación a los grupos. Los análisis estadísticos fueron realizados con el programa SPSS versión 22.0.

Tabla 1: Variables de evaluación

\begin{tabular}{ccc}
\hline Evaluación & Categoría & Variables \\
\hline Diagnóstica $(\mathrm{Ed})$ & Generales & Definición agroforestería \\
\cline { 3 - 3 } & & Saberes tradicionales \\
\cline { 3 - 3 } & & Sistemas agroforestales \\
\cline { 3 - 3 } & Generales & Arreglos agroforestales \\
\hline Posterior $(\mathrm{Ep})$ & & Definición agroforestería \\
\cline { 2 - 2 } & & Saberes tradicionales \\
\cline { 2 - 2 } & & Sistemas agroforestales \\
\cline { 2 - 2 } & & Arreglos agroforestales \\
\hline
\end{tabular}

\section{RESULTADOS Y DISCUSIÓN}

\section{Análisis de relación de aprendizaje adquirido}

A continuación se presentan los resultados obtenidos de las evaluaciones con relación a la media, desviación estándar y error estándar de la media (Tabla 2) 
Tabla 2: Comparación de medias de resultados en evaluaciones entre grupo control y experimental

\begin{tabular}{|c|c|c|c|c|}
\hline Evaluación & Grupo & Media & Desviación típ. & $\begin{array}{l}\text { Error típ. de la } \\
\text { media }\end{array}$ \\
\hline \multirow[t]{2}{*}{ Diagnóstica } & Grupo Experimental & 1,2188 & ,90418 & ,15984 \\
\hline & Grupo Control & 1,3704 & ,86982 & ,16740 \\
\hline \multirow{2}{*}{$\begin{array}{l}\text { General } \\
\text { repetición } 1\end{array}$} & Grupo Experimental & 3,6328 & 1,28418 & ,22701 \\
\hline & Grupo Control & 3,8426 & 1,09225 & ,21020 \\
\hline \multirow{2}{*}{$\begin{array}{l}\text { General } \\
\text { repetición } 2\end{array}$} & Grupo Experimental & 4,4141 & ,83909 & ,14833 \\
\hline & Grupo Control & 4,1204 & ,96916 & ,18652 \\
\hline \multirow{2}{*}{$\begin{array}{l}\text { General } \\
\text { repetición } 3\end{array}$} & Grupo Experimental & 4,4141 & ,83909 & ,14833 \\
\hline & Grupo Control & 4,1204 & ,96916 & ,18652 \\
\hline \multirow{2}{*}{$\begin{array}{l}\text { Especifica } \\
\text { repetición } 1\end{array}$} & Grupo Experimental & 3,0469 & ,93731 & , 16570 \\
\hline & Grupo Control & 2,8704 & 1,06752 & ,20544 \\
\hline \multirow{2}{*}{$\begin{array}{l}\text { Especifica } \\
\text { repetición } 2\end{array}$} & Grupo Experimental & 3,5417 & ,51848 & 09165 \\
\hline & Grupo Control & 3,6111 & ,83333 & ,16038 \\
\hline \multirow{2}{*}{$\begin{array}{l}\text { Especifica } \\
\text { repetición } 3\end{array}$} & Grupo Experimental & 3,5417 & ,51848 & ,09165 \\
\hline & Grupo Control & 3,6111 & ,83333 & ,16038 \\
\hline
\end{tabular}

El promedio de los resultados de las evaluaciones posteriores realizadas al grupo experimental fue de 3,765 y del grupo control de 3,696, indicó un desempeño básico, entendido como la superación de los contenidos de agroforestería.

Los resultados obtenidos de la prueba $T$ de Student para muestras independientes indican que no hay diferencias estadísticamente significativas entre los grupos control y experimental. Resultados similares encontrados en investigaciones realizadas por Castaño et al (2001), Pineda (2008) y Domínguez et al (2011). Las charlas educativas y los recursos TIC, ofrecen distintas formas de trabajar los contenidos y actividades (Cacheiro, 2011); sin embargo, las TIC han permitido pasar de un uso informativo y colaborativo a un uso didáctico para lograr resultados de aprendizaje (Carvajal, 2009; Cacheiro, 2011).

Analizando en conjunto todas las variables en el tiempo, por medio del modelo lineal general con medidas repetidas, se encontró en el efecto principal intrasujetos "evaluaciones generales" diferencias estadísticamente significativas $(P<0,05)(F=299,403 ; g l=1 ; p=0,000)$ y para los resultados intrasujetos con "evaluaciones específicas", mediante el modelo mencionado anteriormente también se encontraron diferencias estadísticamente significativas $(p<0,05)(F=11,08 ; g l=1 ; p=0,02)$ Sin embargo en forma general se atribuye una tendencia en el mejoramiento de resultados de evaluaciones en el tiempo, sin tener en cuenta la herramienta utilizada para la enseñanza; evidenciando el aprendizaje de agroforestería adquirido por los estudiantes. Sumado a esto, el interés y motivación de los estudiantes por la temática de agroforestería fue un factor en común, al ser ésta una idea innovadora en el contexto rural (Somarriba, 2009).

Es importante mencionar que las TIC proporcionan nuevas oportunidades a la educación, a pesar de que el tiempo de evaluación fue corto; Moya et al., (2011), plantean que las TIC llegan a todos los rincones de las sociedades, creando nuevas relaciones y modificando los estilos de vida y de pensamiento; además, ofrecen facilidades para la gestión de la información, flexibilización espacio temporal y posibilidades de interacción entre los usuarios (Mirete y García, 2014; Hernández et al, 2014). Los diversos modelos de extensión rural en Colombia, han optado por involucrar cada vez más los medios de comunicación al desempeñar un papel mediador entre las instituciones generadoras de conocimiento y los actores rurales (Carvajal, 2009), además de contribuir en la disminución de la brecha digital que ha venido impactando en los procesos de enseñanza aprendizaje impartidos por las TIC (Fossatti, 2009; Domínguez et al, 2011).

Las aplicaciones multimedia y el uso de las tecnologías son las principales herramientas que hoy en día han contribuido a la comunidad educativa en el proceso de formación; considerando que los estudiantes se 
muestran interesados por herramientas diferentes a las tradicionales utilizadas por los docentes; además, estas innovaciones permiten trabajar desde el hogar, el aula o puntos digitales establecidos para este fin, desde donde se facilita complementar la clase convencional (Leonel y Luna, 2016).

\section{CONCLUSIONES}

En forma general el aprendizaje agroforestal adquirido por los jóvenes rurales mediante el uso del material didáctico manual y el software educativo "Mundo Agroforestal", facilitó la comprensión de las diferentes temáticas; sin embargo, en el grupo experimental se resalta la motivación e interés por la exploración de las interfaces, convirtiéndolo en un espacio de interacción dinámico donde el usuario puede construir su conocimiento individualmente o con orientación del facilitador, además de compartir con sus compañeros; en este sentido, el software al ser un material contextualizado, contribuye en la consolidación de tejido social y espacios para la construcción de paz.

A pesar de que el tiempo de evaluación fue limitado, el software "Mundo Agroforestal" facilitó la aprehensión del conocimiento; sin embargo, es importante considerar evaluaciones a corto plazo (menor a un año), mediano y largo plazo cada dos años consecutivos a fin de contar con tres periodos de evaluación combinados con variables de evaluación orientadas a la adopción de sistemas agroforestales.

\section{REFERENCIAS}

Bossio, J. y otros tres autores, Desarrollo rural y tecnologías de la información y comunicación, $1^{\text {a }}$ Edición, GTZ, ITDG, MINAG-DGIA, Lima, Perú (2005)

Builes, C., "Kioskos Vive Digital": Una estrategia digital y de conectividad para promover la inclusión social en comunidades rurales de Colombia, International Journal of Psychological Research, 9(1), 126-130 (2016)

Cacheiro, M., Recursos educativos TIC de información, colaboración y aprendizaje, Revista de Medios y Educación, 39(1), 69-81 (2011)

Carvajal, V., Inclusión o exclusión social: el reto de las TIC y el caso de las poblaciones rurales centroamericanas. Apuntes alrededor de la experiencia, Revista Científica de la Fundación Iberoamericana para la Excelencia Educativa, 2(5), 44-64 (2009)

Castaño, G., J. Convers e I. Solarte, Estudio aleatorizado comparativo entre la enseñanza tradicional vs el uso de computadores e internet, en el curso de Farmacología para estudiantes de pregrado, Revista Universitas Médica, 42(4), 9-18 (2001)

Clavera, T. y otros 4 autores, Elaboración de software educativo para la asignatura Introducción a la Estomatología Integral, Revista Habanera de Ciencias Médicas, 14(4), 506-515 (2015)

Cova, A., X. Arrieta y V. Riveros, Análisis y comparación de diversos modelos de evaluación de software educativo, Revista Venezolana de Información, Tecnología y Conocimiento, 5(3), 45-67 (2008)

Cox, M., y otros cinco autores, Researching IT in education, Journal of Computer Assisted Learning, 29(1), 474-486 (2013)

De la Torre, L. y J. Domínguez, Las TIC en el proceso de enseñanza aprendizaje a través de los objetos de aprendizaje, Revista Cubana de Informática Médica, 4(1), 91-100 (2012)

Domínguez, E. y otros cuatro autores, El ABP mediado con tecnología móvil como estrategia pedagógica para el desarrollo de la competencia matemática en resolución de problemas: un caso con la adición de números enteros negativos, Revista Zona Próxima, 14(1), 1-10 (2011)

Fossatti, M., Tecnologías de Información y Comunicación en el Desarrollo Rural: un marco para la reflexión, Revist@ IICA, 1(1), 55-71 (2009)

Gómez, U., J. Pérez y J. Ramírez, Sistema de información agrícola para la diminución de brechas entre oferta y demanda - AGROCRAFT, Información Tecnológica, 27(3), 215-220 (2016)

Hernández, R., C. Fernández y P. Baptista, Metodología de la investigación, 4a edición, McGraw-Hill Interamericana, Ciudad de México, México (2007) 
Hernández, M. y otros tres autores, Las Tecnologías de la Información y la Comunicación (TICs) en la Enseñanza-Aprendizaje de la Química Orgánica a través de Imágenes, Juegos y Videos, Revista Formación Universitaria, 7(1), 31-40 (2014)

Ledo, M., F. Martínez y A. Ruiz, Software Educativo, Educación Médica Superior, 40(1), 97-110 (2010)

León, A., C. Luna y H. Leonel, Software Educativo "Mundo Agroforestal". Estudio de caso, Subcuenca Alta del Río Pasto, Nariño, Colombia, Revista de Ciencias Agrícolas, 31(1), 65-77 (2014)

Leonel, H., y C. Luna, Herramientas didácticas para la formación agroforestal, $1^{\text {a }}$ edición, Universidad de Nariño, San Juan de Pasto, Colombia (2016)

Mirete, A., y F. García, Rendimiento académico y TIC una experiencia con webs didácticas en la Universidad de Murcia, Revista de Medios y Educación, 44(1), 169-183 (2014)

Moya, M., y otros tres autores, Análisis de los estilos de aprendizaje y las TIC en la formación personal del alumnado universitario a través del cuestionario REATIC, Revista de Investigación Educativa, 29(1), 137-156 (2011)

Pineda, C., Rendimiento académico obtenido con un aplicativo multimedial vs clase tradicional, Revista U.D.C.A Actualidad \& Divulgación Científica, 11(1), 57-65 (2008)

Saidu, A., A. Tukur, y S. Adamu, Promoting sustainable development through ict in developing countries, European Journal of Computer Science and Information Technology, 2(2), 24-29 (2014)

Somarriba, E., Planificación agroforestal de fincas, $1^{\text {a }}$ edición, CATIE-GTZ, Turrialba, Costa Rica (2009) 